\title{
$\beta$-Lactam Allergy and Cross-Reactivity: A Clinician's Guide to Selecting an Alternative Antibiotic
}

This article was published in the following Dove Press journal:

Journal of Asthma and Allergy

\author{
Cristiano Caruso ${ }^{1}$ \\ Rocco Luigi Valluzzi ${ }^{2}$ \\ Stefania Colantuono' \\ Francesco Gaeta' \\ Antonino Romano 3,4 \\ 'Allergy Unit, Columbus Hospital, \\ Fondazione Policlinico A. Gemelli, \\ IRCCS, Rome, Italy; ${ }^{2}$ Department of \\ Pediatrics, Division of Allergy, Pediatric \\ Hospital Bambino Gesù, Rome, Vatican \\ City, Italy; ${ }^{3}$ Oasi Research Institute- \\ IRCCS, Troina, Italy; ${ }^{4}$ Fondazione \\ Mediterranea G.B. Morgagni, Catania, \\ Italy
}

\begin{abstract}
Lactams which include penicillins, cephalosporins, carbapenems, and monobactams are the most common antibiotic classes reported to cause allergic reactions to drugs. This review is mainly about published studies assessing the cross-reactivity among $\beta$-lactams in penicillin- or cephalosporin-allergic subjects by carrying out diagnostic tests with alternative $\beta$-lactams and, if appropriate, graded challenges. Several studies demonstrated that cross-reactivity connected with the $\beta$-lactam ring, causing positive responses to allergy tests with all $\beta$-lactams, is infrequent in subjects with an IgE-mediated allergy and anecdotal in those with a T-cell-mediated allergy. Identities or similarities of $\beta$ lactam side-chain structures are mainly responsible for cross-reactivity among these antibiotics. For example, in aminopenicillin-allergic subjects, cross-reactivity with aminocephalosporins could possibly be over 30\%. On the other hand, in a few prospective studies of penicillin-allergic individuals, less than $1 \%$ of cases show a cross-reactivity between penicillins and both aztreonam and carbapenems. Particular patterns of allergytest positivity observed in some studies that assessed cross-reactivity among $\beta$-lactams seem to indicate that prior exposures may be responsible for coexisting sensitivities. Therefore, pre-treatment skin tests with the related $\beta$-lactams are suggested before administering them via graded challenges to $\beta$-lactam-allergic patients who need alternative $\beta$-lactams.
\end{abstract}

Keywords: aztreonam, $\beta$-lactams, carbapenems, cephalosporins, cross-reactivity, hypersensitivity, penicillins, skin tests

\section{Introduction}

$\beta$-Lactams which include penicillins, cephalosporins, carbapenems, and monobactams, are the most common antibiotic classes reported to cause allergic reactions to drugs. All $\beta$-lactams share a 4 -membered $\beta$-lactam ring. In penicillins, it is attached to a 5-membered thiazolidine ring; the side chain $(\mathrm{R})$ differentiates the penicillins (Figure 1). Instead of the 5-membered thiazolidine ring of penicillins, cephalosporins have a 6-membered sulfur-containing dihydrothiazine ring and 2 side chains (R1 and R2), which distinguish the different compounds (Figures 1, 2A and B). Carbapenems (eg, imipenem, meropenem, ertapenem, and doripenem) contain a carbon double bond instead of sulfur in the 5-membered thiazolidine ring and have a side chain (R), which distinguishes the different carbapenems (Figure 3). Aztreonam is the only monobactam commercially available; it contains only the $\beta$-lactam ring (Figure 3 ).

Penicillins and cephalosporins are frequently responsible for hypersensitivity reactions (HSRs). ${ }^{1}$ IgE-mediated ones occur within 6 hours after the last drug administration (ie, immediate reactions), though typically occur within one hour
Correspondence: Cristiano Caruso Tel +393348079 I 33 Email cristiano.caruso@policlinicogemelli. it 


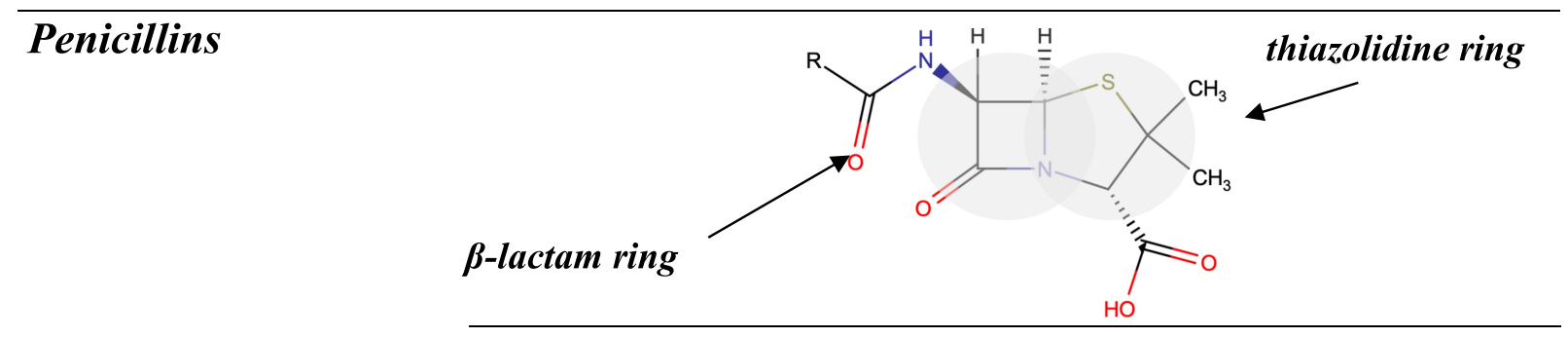

$\mathbf{R}$ side chain
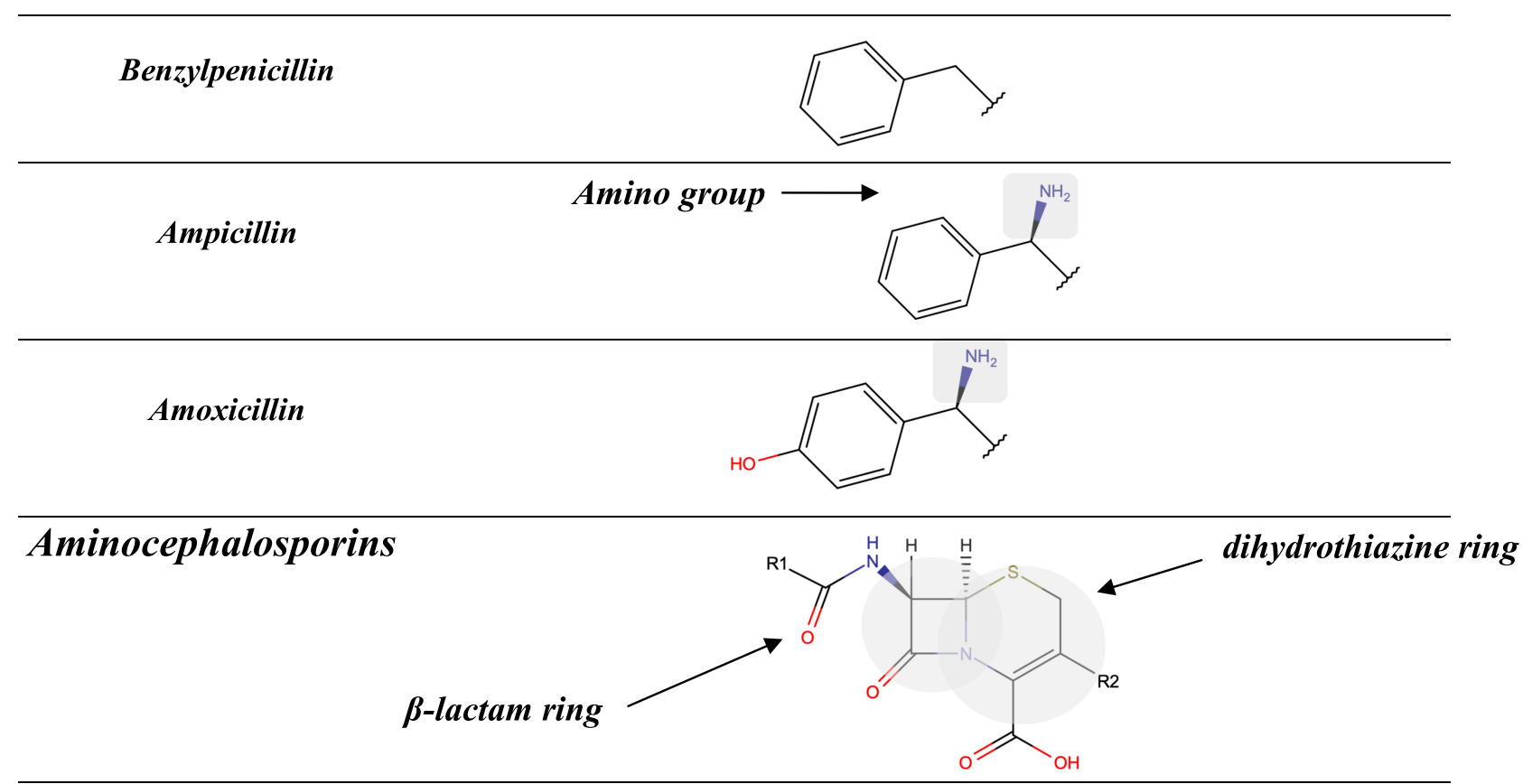

\section{R1 side chain}

Cefadroxil

Figure I Chemical structures of penicillins, with the amino group of ampicillin and amoxicillin, as well as that of cephalosporins of group B (ie, aminocephalosporins) highlighted in gray.

of the first dose of a new treatment course. ${ }^{2-4}$ These reactions are characterized by a silent sensitization, with a transient mast-cell unresponsiveness to the initial administration of the drug. ${ }^{5}$ They usually manifest as cutaneous (eg, itching, hives, angioedema, generalized erythema), respiratory (eg, nasal congestion, rhinorrhea, 
A

\section{Cephalosporins}

B-lactam ring

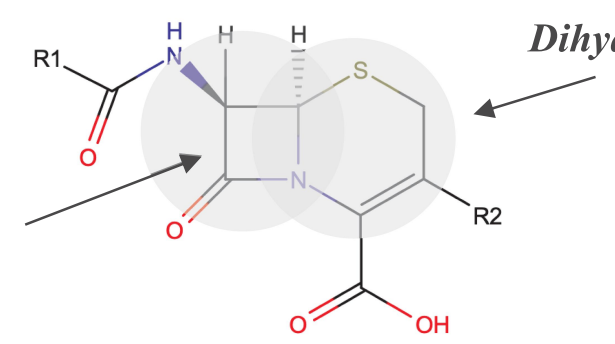

Dihydrothiazinic ring

R1 side chain

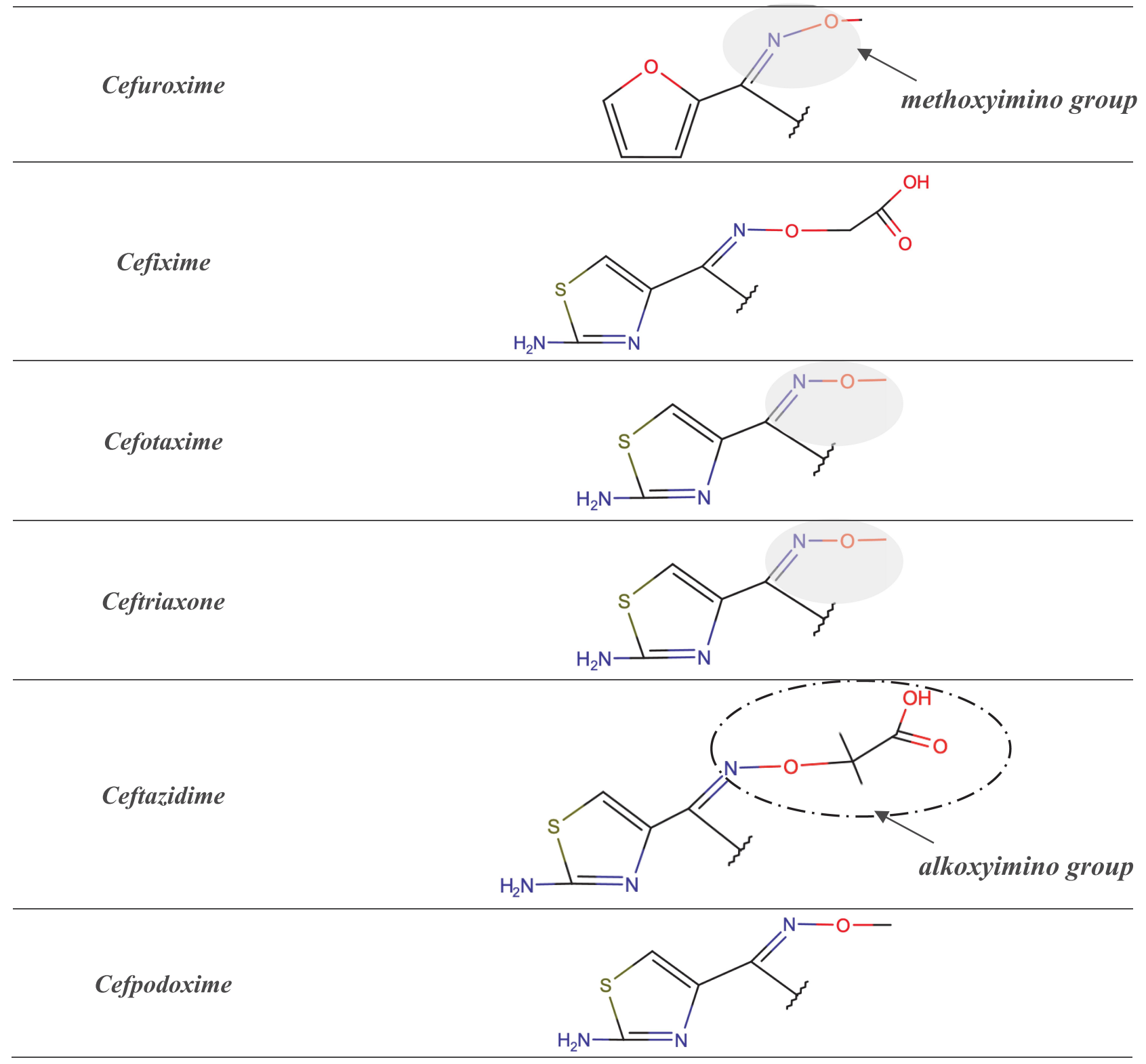

Figure 2 Continued. 
B

\section{Cephalosporins}

$\beta$-lactam ring

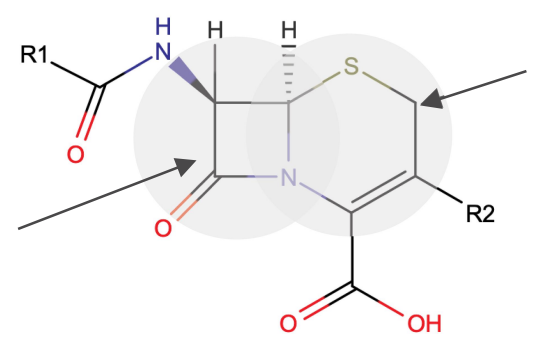

Dihydrothiazinic ring

\section{R1 side chain}

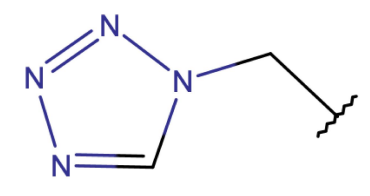

R2 side chain

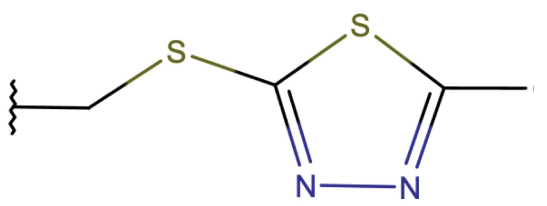

$\mathrm{N}-\mathrm{N}$

Cefazolin

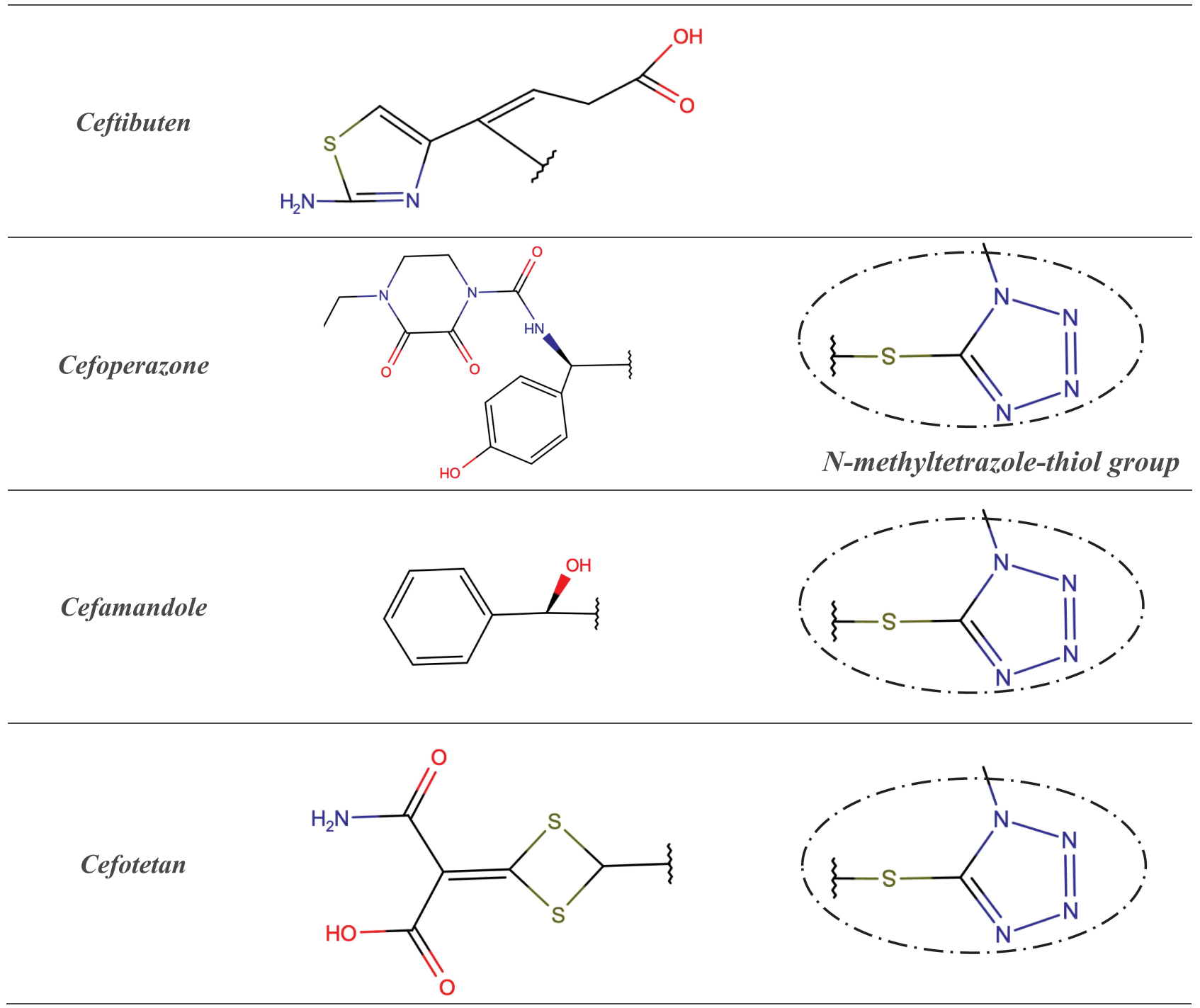

Figure 2 (A and B) Chemical structures of cephalosporins other than aminocephalosporins, with the methoxyimino group of cephalosporins of group A highlighted in gray and the alkoxyimino group of ceftazidime and $\mathrm{N}$-methyltetrazole-thiol group of cefamandole and cefoperazone circled in black. 


\section{Carbapenems}

\section{$\beta$-lactam ring}
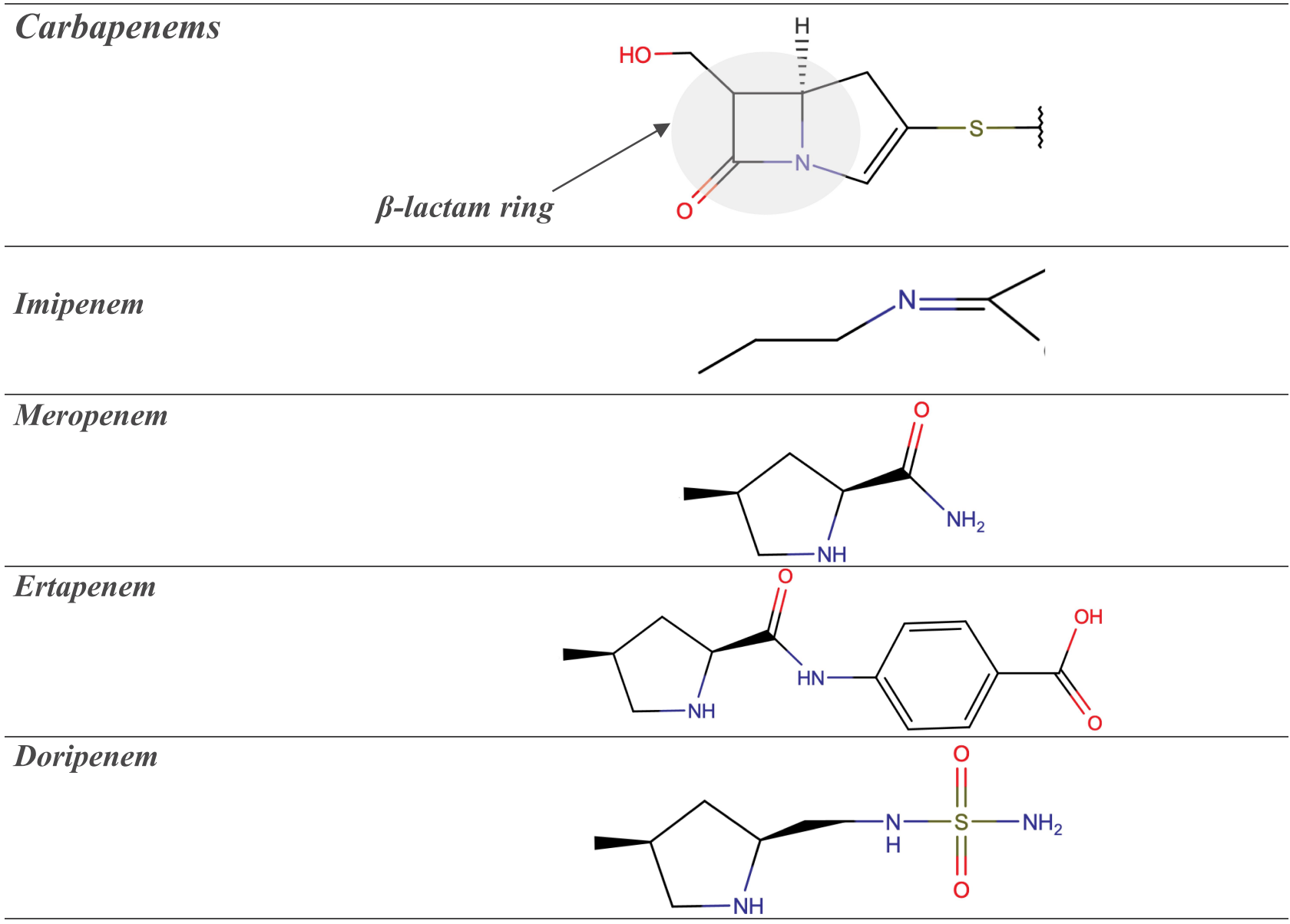

\section{Aztreonam}

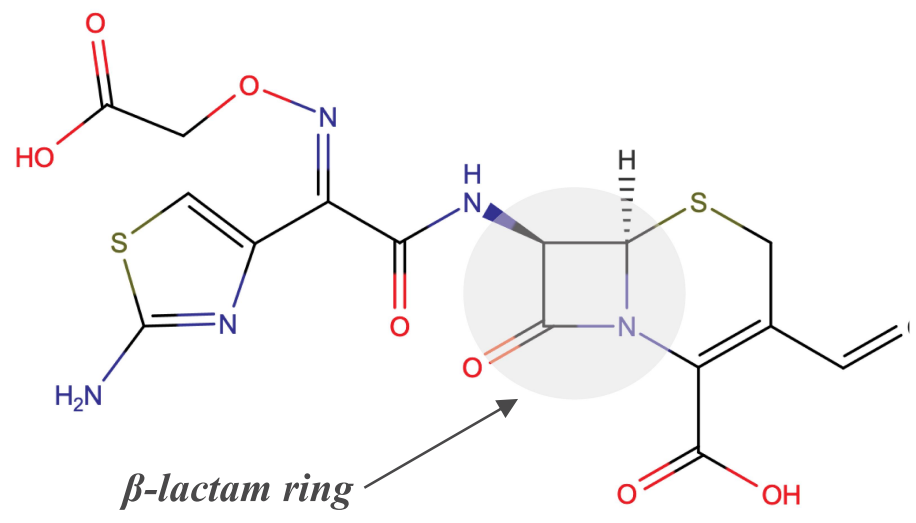

Figure 3 Chemical structures of carbapenems and aztreonam.

sneezing, hoarseness, cough, wheezing), gastrointestinal (eg, nausea, diarrhea, mild abdominal pain, vomiting), cardiovascular (eg, tachycardia, hypotension) symptoms, which may appear isolated or in combination as in anaphylaxis. ${ }^{4}$ T-cell-mediated HSRs may occur at any time from 1 hour after the first drug administration (ie, nonimmediate reactions), commonly after many days of treatment. ${ }^{3}$ In this case, cytotoxic and cytokine-secreting $\mathrm{T}$ cells orchestrate the inflammatory cells (ie, $\mathrm{T}$ cells, PMN, eosinophils). ${ }^{5}$ Maculopapular exanthema (MPE) and delayed-appearing urticaria are the most frequent nonimmediate reactions. ${ }^{3,6}$ Severe cutaneous adverse reactions 
(SCARs), ie acute generalized exanthematous pustulosis (AGEP), Stevens-Johnson syndrome (SJS), toxic epidermal necrolysis (TEN), and drug-induced hypersensitivity syndrome or drug rash with eosinophilia and systemic symptoms (DRESS), are the most serious expressions of $\beta$-lactam nonimmediate reactions.

The $\beta$-lactam ring, the thiazolidine/dihydrothiazine rings, and the side-chains can all sensitize subjects treated with $\beta$ lactams. In particular, side chains contribute significantly to immunological recognition and therefore the structures are most frequently responsible for allergic cross-reactivity. ${ }^{1,7-12}$

In $\beta$-lactam-allergic patients, the diagnostic workup with alternative drugs shows the cross-reactivity and, above all, allows to treat patients with safe drugs. ${ }^{4,13}$

This review is mainly about prospective studies which assessed the cross-reactivity among $\beta$-lactams in penicillin- or cephalosporin-allergic subjects by carrying out in vivo tests and, if available, in vitro ones with alternative $\beta$-lactams and, in case of negative results, administering them via graded challenges.

\section{Selecting Alternative $\beta$-Lactams in $\beta$-Lactam-Allergic Subjects}

In subjects reporting HSRs to $\beta$-lactams, the two main goals of the diagnostic workup are to confirm or exclude allergy to the $\beta$-lactam concerned and, in case of allergy diagnosis, to find safe alternatives, particularly among other $\beta$-lactams. ${ }^{4}$ An accurate clinical history is crucial for the assessment of subjects reporting HSRs to $\beta$-lactams, followed by skin tests (STs) and/or patch tests (PTs). In selected cases presenting negative results, challenges with the suspected $\beta$-lactams can be considered for the final diagnosis. ${ }^{4}$ Subjects with suspected IgE-mediated reactions are evaluated by immediatereading skin prick tests and intradermal tests (IDTs) by immediate-reading are performed in subjects with suspected IgE-mediated reactions, whereas those with nonimmediate reactions by delayed-reading STs and/or PTs., ${ }^{4,14}$

In vitro tests can be used as a complement to the above diagnostic tests. The main in vitro tests for assessing subjects with immediate reactions to $\beta$-lactams are the serum specific IgE (sIgE) assay and the basophil activation test. The lymphocyte transformation test and the enzymelinked immunosorbent spot assay can be used for evaluating subjects with nonimmediate reactions. ${ }^{4}$

There are few prospective studies of $\beta$-lactam-allergic subjects which assessed cross-reactivity among $\beta$-lactams by carrying out allergy tests with $\beta$-lactams other than those responsible and, in case of negative results, by performing graded challenges with them. ${ }^{4-9-12}$

\section{Penicillin-Allergic Subjects}

Individuals with histories of penicillin allergy are more likely to receive alternative broad-spectrum antibiotics, including vancomycin and quinolones, which can lead to higher costs, prolonged hospitalizations, and elevated number of infection with methicillin-resistant Staphylococcus aureus, vancomycin-resistant Enterococcus, and Clostridioides difficile (formerly Clostridium difficile). ${ }^{15-17}$ Although a large number of patients are labeled as allergic to penicillin, more than $95 \%$ of them can tolerate penicillin after an appropriate evaluation. ${ }^{18}$ Due to the low prevalence of true penicillin allergy and the disadvantage from using alternative antibiotics, all subjects who report HSRs to penicillins should be evaluated in order to confirm or exclude a penicillin-allergy diagnosis. ${ }^{19,20}$

\section{Selecting Alternative Penicillins}

Several studies found a greatness of cross-reactivity between benzylpenicillin (penicillin G [PG]) and semisynthetic penicillins, as well as among the latter, in particular aminopenicillins (ie, amoxicillin, ampicillin, bacampicillin, and pivampicillin) which share an amino group in their side chain (Figure 1). ${ }^{9-12}$ However, there are studies in which participants with either an IgE-mediated ${ }^{21}$ or a T-cell-mediated allergy to aminopenicillins ${ }^{22,23}$ showed negative results at in vivo tests with PG and/or phenoxymethylpenicillin (penicillin V [PV]) and tolerated graded challenges with them. Specifically, Blanca-Lopez et $\mathrm{al}^{21}$ diagnosed hypersensitivity in 58 subjects reporting immediate reactions to amoxicillin or amoxicillin/clavulanic acid. Of these 58 individuals, 7 were positive to $P G$ determinants, 40 were positive to amoxicillin and tolerated both PG and PV, and the remaining 11 were positive only to clavulanic acid and tolerated PG, PV, and amoxicillin.

In two studies of more than 30 subjects with a T-cellmediated allergy to aminopenicillins, ${ }^{22,23}$ the rate of crossreactivity to $\mathrm{PG}$ and/or PV was $9.1 \%$ and $28.2 \%$, respectively. Specifically, in a study of ours, ${ }^{22} 33$ of the 60 participants reporting MPEs associated with aminopenicillin therapy were positive to PTs and delayed-reading IDTs with ampicillin and amoxicillin, and 3 also to those with PG; 17 subjects negative to PG reagents accepted challenges with PV and tolerated them. In another study, ${ }^{23}$ of the 71 participants with a delayed allergy to aminopenicillins, 16 presented positive PTs or IDTs to both PG and 
PV and 4 only to PG. All 51 subjects who underwent challenges with PV tolerated them.

Concerning subjects allergic to penicillins other than aminopenicillins, in a recent study by Kennard et al, ${ }^{24} 4$ patients with an immediate hypersensitivity to flucloxacillin and one with a delayed hypersensitivity to it tolerated challenges with amoxicillin and PG, respectively, found negative in allergy tests.

\section{Selecting Cephalosporins}

The data concerning the measure of cross-reactivity betwixt penicillins and cephalosporins differ markedly and are frequently inaccurate. ${ }^{25} \mathrm{~A}$ meta-analysis of articles published between 1966 and 2005, which compared HSRs to cephalosporins in penicillin-allergic and nonpenicillin-allergic patients, showed a considerable rise (odds ratio $=4.8$ ) in allergic reactions to all firstgeneration cephalosporins plus cefamandole, but no increment with second- or third-generation. ${ }^{26}$ Nonetheless, the adulteration of these early cephalosporins with trace amounts of PG it may have leaded to an overset of the degree of cross-reactivity between these $\beta$-lactams. Furthermore, this meta-analysis ${ }^{26}$ comprehended retrospective studies, in which the diagnosis of penicillin allergy was based only on the clinical history. A recent meta-analysis $^{12}$ of 21 studies on cephalosporin crossreactivity performed between January 1980 and December 2016, which included 1269 penicillin-allergic patients, showed that the rate of cross-reactivity varied with the degree of similarity between R1 side chains. In effect, such risk was $16.5 \%$ for aminocephalosporins (ie, cephalexin, cefadroxil, cefprozil, and cefaclor), which share an identical side chain (similarity score $=1$ ) with an aminopenicillin, 5.6\% for cephalothin, cephaloridine, and cefamandole, which have an R1 side chain with a similarity score of around 0.6 compared with that of PG, and $2.1 \%$ for all those (ie, cefazolin, cefuroxime, cefixime, cefotaxime, ceftriaxone, ceftazidime, cefpodoxime, ceftibuten, and cefepime) with low similarity scores (below 0.4), irrespective of cephalosporin generation (Figures 1, 2A and B).

Note that this meta-analysis ${ }^{12}$ included studies on at least 10 subjects (children and adults) with a documented penicillin allergy (IgE- or T-cell-mediated). In studies carried out since 1990 on more than 25 subjects with proven IgE-mediated allergy to penicillins, ${ }^{27-34}$ the rate of positive cephalosporin STs ranged from $0 \%^{28,30}$ to $33.3 \%$ (Table 1). ${ }^{31}$ The highest rate was found in the study of ours, ${ }^{31}$ in which participants underwent STs with a panel of 9 cephalosporins, including 3 aminocephalosporins (ie, cephalexin, cefadroxil, and cefaclor) that share similar or identical side-chain determinants with the aminopenicillins that were responsible for HSRs in $96 \%$ of the 252 patients assessed. In the aforesaid studies, ${ }^{27-34} 821$ penicillinallergic subjects negative to cephalosporin STs underwent a total of 1825 graded challenges with the related cephalosporins; only 11 challenges $(0.6 \%)$ were positive. Specifically, there were 7 positive challenges $(1.9 \%)$ out of 366 performed with aminocephalosporins, and 4 positive challenges $(0.3 \%)$ out of 1459 carried out with cephalosporins like cefazolin, cefuroxime, ceftriaxone, and ceftazidime that have side chains different from those of penicillins. In other studies, ${ }^{35-38}$ penicillin-allergic subjects underwent challenges or treatments with cephalosporins, such as cephalexin, cefadroxil, cefamandole, and ceftriaxone, without previous STs with the related cephalosporins. The rate of positive responses to cephalosporin challenges ranged from $5.6 \%$ ( 5 of 85$)^{38}$ to $38 \%$ ( 8 of 21). ${ }^{37}$ The highest rate of cephalosporin positive challenges was observed in the study by Miranda et al, ${ }^{37}$ who administered cefadroxil to 21 subjects allergic to amoxicillin.

All this should end the debate on the usefulness of performing STs with cephalosporins before giving them to penicillin-allergic patients, which has also recently taken place. ${ }^{39}$

To be noted that in a study concerning the crossreactivity and tolerability of cefazolin and ceftibuten in 131 penicillin-allergic patients, ${ }^{33}$ one participant was ST positive to all reagents tested, including carbapenems and aztreonam, which indicates a sensitivity to an antigenic determinant of the $\beta$-lactam ring.

With regard to T-cell-mediated allergy, of the 3 studies $^{23,40,41}$ that evaluated cross-reactivity with cephalosporins in adults with such allergy - by carrying out STs and/or PTs with cephalosporins and, in case of negative results, challenges with all tested cephalosporins $-2^{40,41}$ found a rate of cross-reactivity with aminocephalosporins of $19.1 \%$ and $31.2 \%$, respectively. Specifically, in an aforementioned study, ${ }^{41} 214$ consecutive adults with proven T-cell-mediated hypersensitivity to penicillins underwent STs with cephalexin, cefaclor, cefadroxil, cefuroxime, and ceftriaxone. Most subjects had experienced MPEs, whereas 5 had had a TEN, and 2 an AGEP (one of the latter had experienced 2 episodes). All participants were negative to STs with cefuroxime and 


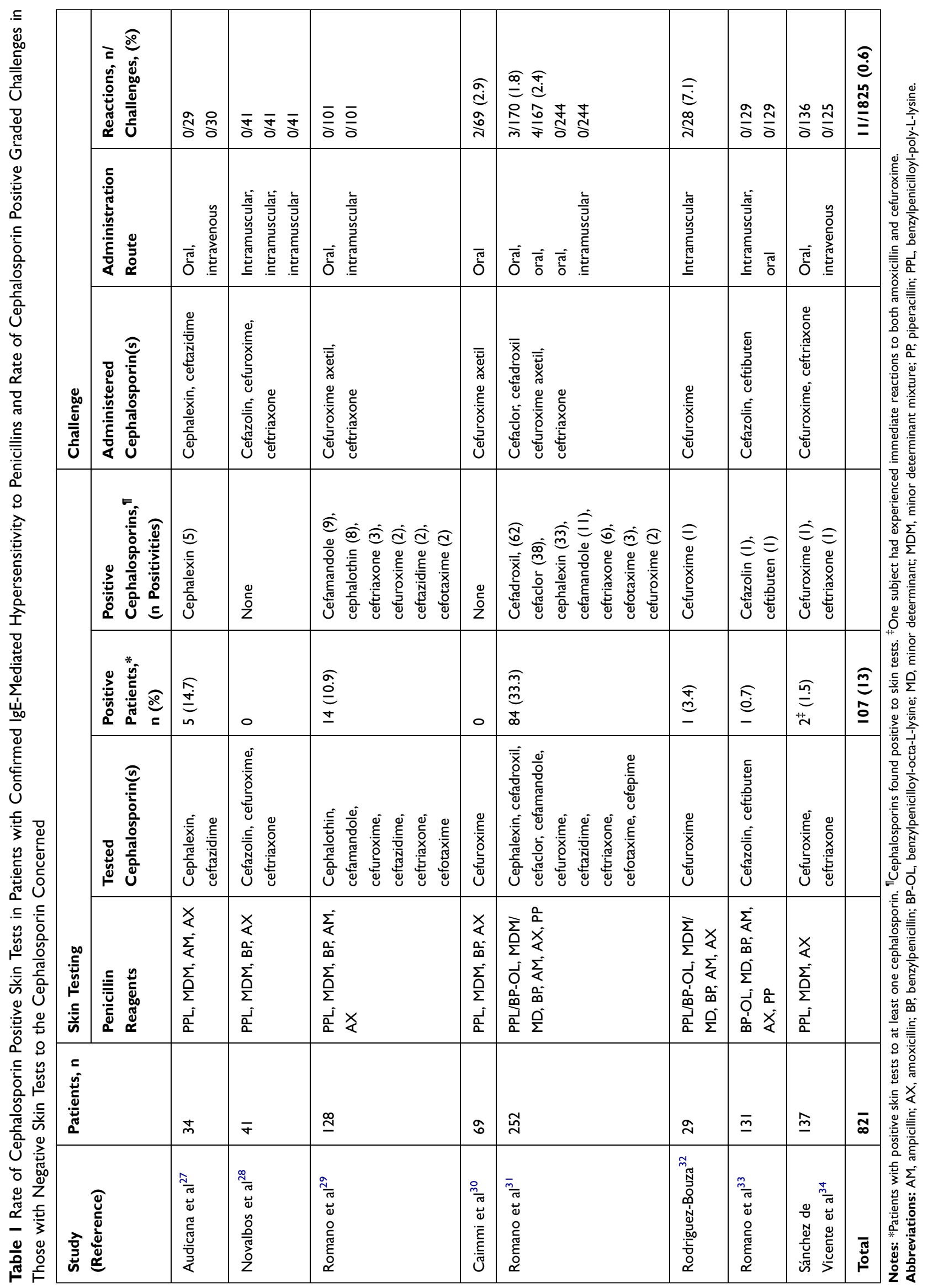


ceftriaxone and tolerated challenges with them. Forty $(18.7 \%)$ of the 214 participants were positive to aminocephalosporin STs (Table 2). Two of these 40 participants had experienced an AGEP and 2 a TEN. Of the 174 participants negative to aminocephalosporin STs, 170 accepted challenges; one reacted to cefaclor. Challenges with aminocephalosporins found negative in skin testing were not carried out in 3 subjects with TEN as index reaction. In the aforementioned studies, ${ }^{23,40,41}$ only 3 $(0.3 \%)$ out of 1083 cephalosporin challenges were positive (Table 2).

In penicillin-allergic patients, therefore, skin testing with cephalosporins - such as cefazolin, cefuroxime, ceftazidime, cefpodoxime, cefixime, ceftriaxone, and ceftibuten that have side chains different from those of penicillins - followed, in case of negative results, by graded challenges has proved to be a safe method to administer these alternative $\beta$-lactams. ${ }^{4,9}$ Note that this approach is the one recommended by both the European Academy of Allergy and Clinical Immunology ${ }^{4,13}$ and the British Society for Allergy and Clinical Immunology. ${ }^{8}$ It is also one of three options in the US practice parameter ${ }^{42}$ concerning cephalosporin administration to patients with histories of penicillin allergy.

In graded challenges, usually, an initial dose of one tenth of the maximum single unit dose (MSUD) is administered and, in case of a negative result, 1 hour later a full MSUD.

In patients with mild/moderate nonimmediate reactions to penicillins who require a cephalosporin, if there's no time to wait for the delayed reading of pre-treatment STs, giving a full dose of a structurally non-related cephalosporin under close surveillance can be considered, as the increased risk does not concern an immediate reaction, such as anaphylaxis, but that of an exanthema recurrence. In a study by Blumenthal et al, ${ }^{43} 17$ patients treated with nafcillin were switched to cefazolin because of non-IgEmediated HSRs, which included MPE $(\mathrm{n}=10)$, immunemediated nephritis $(\mathrm{n}=3)$, isolated eosinophilia $(\mathrm{n}=2)$, immune-mediated hepatitis $(\mathrm{n}=1)$, and a serum sicknesslike reaction $(\mathrm{n}=1)$. All but one patient $(94.1 \%)$ who switched to cefazolin tolerated a therapeutic course with it.

Patients with histories of SCARs associated with penicillin treatments should generally avoid all penicillins and cephalosporins, especially those structurally related to the responsible penicillin, because of the severity of the reported reaction and the not yet well defined sensitivity of allergy tests. Exceptions are possible only after consultation with an allergist experienced in drug allergy. In a study by Trubiano et $\mathrm{al}^{44} 6$ patients with

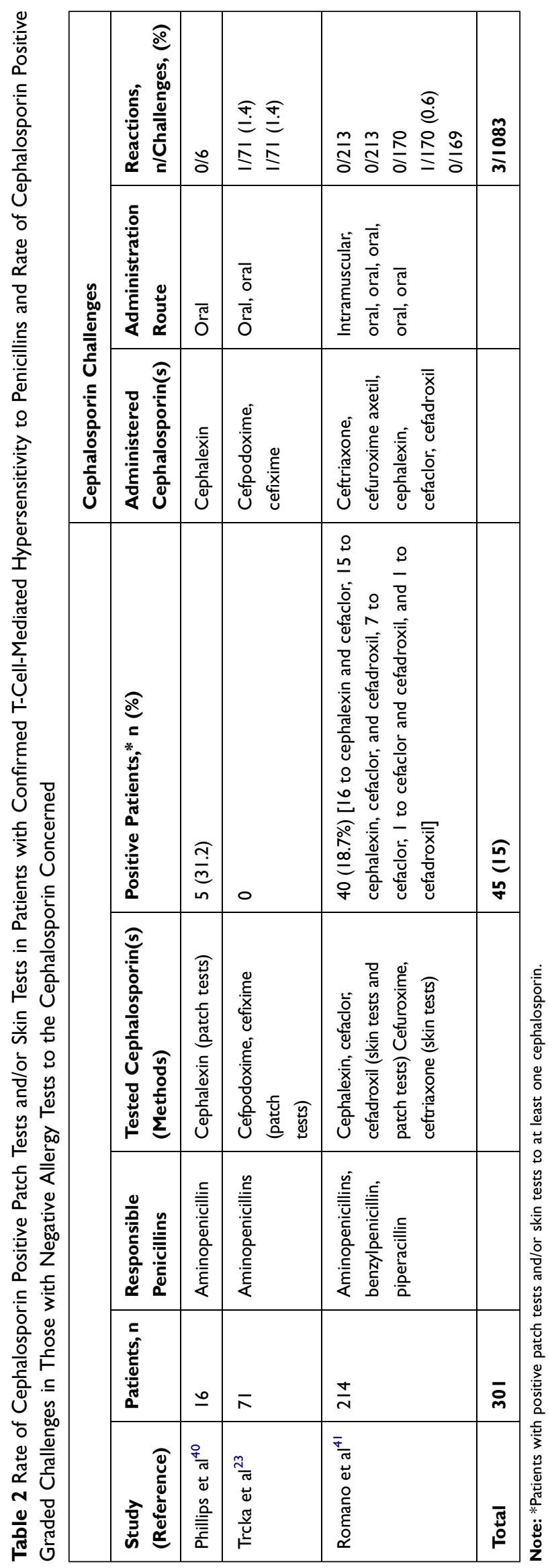


SCARs ( 3 with DRESS and 3 with AGEP) associated with penicillins ( 3 with piperacillin/tazobactam, 2 with flucloxacillin, and 1 with amoxicillin) underwent delayed-reading IDTs with a panel of penicillins and cephalosporins. All were positive to PG, ampicillin, and flucloxacillin; the 3 subjects who had reacted to piperacillin/tazobactam were also positive to this combination. All 6 subjects were negative to cefazolin and ceftriaxone; 5 of them underwent oral challenges with cephalosporins ( 3 with cephalexin and 2 with cefuroxime) and tolerated them.

In a recent retrospective study, ${ }^{45} 13$ of the 29 subjects with SCARs (3 with DRESS and 10 with AGEP) were positive to allergy tests with the responsible $\beta$-lactams -9 to amoxicillin, 1 to cloxacillin, 1 to cefoxitin, 1 to cefuroxime, and 1 to ceftriaxone (11 to PTs and 2 to delayedreading IDTs) - and underwent PTs and STs with a panel of reagents which included penicillins, cephalosporins, aztreonam, and carbapenems. One of the 2 patients with DRESS and 5 of those with AGEP associated with amoxicillin were also positive to other penicillins. Only one subject who had reacted to both amoxicillin and cefpodoxime was positive to a cephalosporin (ie, cefotaxime). In this study, ${ }^{45}$ however, unlike in aforementioned studies, ${ }^{41,44}$ penicillin-allergic subjects negative to allergy tests with cephalosporins did not undergo challenges with them.

\section{Selecting Carbapenems or Aztreonam}

Until 15 years ago, carbapenems were considered potentially dangerous for individuals with penicillin allergy ${ }^{46}$ because in a study by Saxon et $\mathrm{al}^{47} 9$ (47.4\%) out of 19 subjects with such allergy were ST positive to imipenem and/or its metabolites. Subsequent prospective studies, ${ }^{48-52}$ each performed on more than 100 subjects with a well-demonstrated IgE-mediated penicillin allergy, found a rate of cross-reactivity between penicillins and carbapenems lower than $1 \%$ by carrying out STs with different compounds (ie, imipenem/cilastatin, meropenem, and ertapenem).

Regarding aztreonam, in a study of ours, ${ }^{52} 212$ consecutive adults with proven IgE-mediated allergy to penicillins, mostly aminopenicillins, displayed negative STs to aztreonam.

In the aforementioned studies, ${ }^{48-52}$ all participants tolerated challenges with the alternative $\beta$-lactams found negative in skin testing, including 211 subjects who underwent aztreonam challenges.
According to a recent review on penicillin allergy, ${ }^{53}$ patients with reported immediate reactions to penicillin have a $<1 \%$ risk of IgE-mediated cross-reactivity to carbapenems and $0 \%$ to monobactams. However, in a study that assessed the tolerability of meropenem in 104 subjects with an IgE-mediated penicillin allergy, ${ }^{49} 1$ participant $(0.9 \%)$ presented a positive reaction to the IDT with meropenem and had positive results to all penicillin reagents in both in vivo and in vitro tests, as well to STs with imipenem/cilastatin and cephalosporins. Therefore, this subject probably had IgE antibodies to an antigenic determinant of the common $\beta$-lactam ring like the one described above. ${ }^{33}$

With regard to the cross-reaction between penicillins and either carbapenems or aztreonam in subjects with a nonimmediate hypersensitivity, the literature data are less robust. Two studies regarding more than 200 subjects ${ }^{41,54}$ presented no cross-reactivity with these alternative $\beta$-lactams. In particular, all patients were ST negative to carbapenems (ie, imipenem/cilastatin, meropenem, and ertapenem) and aztreonam and tolerated challenges with the alternative $\beta$-lactams concerned. Considering the results of a study of ours, ${ }^{54}$ the positive responses to PTs with imipenem/cilastatin previously observed by Schiavino et al ${ }^{55}$ in 4 of 73 subjects with a delayed penicillin allergy appear unreliable. In fact, it seems unlikely that all 4 patients of the aforesaid study ${ }^{55}$ positive to imipenem/cilastatin PTs were negative to delayed-reading IDTs, which are more sensitive than PTs. ${ }^{6}$ Moreover, Schiavino et $\mathrm{al}^{55}$ found 2 PT positivities to benzylpenicilloyl-poly-L-lysine (PPL). However, as noted by Levine ${ }^{56}$ in delayed reactions to penicillins, polylysine is a nonimmunogenic carrier. Therefore, the positivity to PTs with imipenem/cilastatin may have been false, as that to PTs with PPL.

Concerning subjects with SCARs, in the study by Bérot et $\mathrm{al}^{45}$ none of the 13 patients with such reactions and positive allergy tests with the responsible $\beta$-lactams was positive to aztreonam and carbapenems. In this study, ${ }^{45}$ subjects displaying negative results in allergy tests with these alternative $\beta$-lactams did not undergo challenges with them, whereas in a study of ours, ${ }^{41} 7$ subjects with a T-cell-mediated hypersensitivity to aminopenicillins (5 with TEN and 2 with AGEP) tolerated challenges with aztreonam found negative in skin testing. However, Fernando ${ }^{57}$ described a case that reported a history of a generalized rash associated with PG and cephalexin, respectively, as well as that of an AGEP 
associated with ertapenem. He underwent patch testing with an unspecified reagent panel and was positive at 48 hours to PG, cephalothin, meropenem, and ertapenem. Moreover, Sameed et $\mathrm{al}^{58}$ reported a case of meropeneminduced SJS/TEN in a patient with a history of SJS from amoxicillin. This patient did not undergo allergy evaluation. Therefore, a concomitant sensitization to different $\beta$ lactams cannot be excluded in these 2 subjects.

\section{Cephalosporin-Allergic Subjects}

HSRs to cephalosporins are becoming increasingly common, with approximately $1-3 \%$ of the population reporting them. ${ }^{11}$ In particular, cephalosporins are one of the leading causes for perioperative anaphylaxis and SCARs. ${ }^{59}$ Even though STs with cephalosporins are not as well validated as those with penicillins, ${ }^{7,42,59}$ studies carried out on at least 20 subjects demonstrated that they are reliable and effective for diagnosing both immediate $e^{60-65}$ and delayed hypersensitivity ${ }^{66}$ to these $\beta$-lactams. Of note, in a Korean study, ${ }^{67} 74(5.2 \%)$ of 1421 participants who underwent preoperative cephalosporin STs were positive to at least one cephalosporin. However, all 74 subjects tolerated a challenge dose of the same or different cephalosporin found positive in skin testing.

\section{Selecting Penicillins}

In subjects with proven IgE-mediated allergy to cephalosporins, a few studies assessed the cross-reactivity between cephalosporins and the other classes of $\beta$-lactams by performing graded challenges with alternative $\beta$-lactams found negative in allergy tests. In a study by Antunez et al, ${ }^{61} 2$ of 24 cephalosporin-allergic subjects were ST positive to penicillin reagents, while 22 were ST negative to them and tolerated PG challenges. In a study of 98 subjects with proven cephalosporin allergy who underwent sIgE assays and STs with penicillin reagents as well as STs with carbapenems and aztreonam, ${ }^{68} 25$ participants (25.5\%) had positive allergy tests to penicillins. All 73 subjects negative to penicillin reagents tolerated amoxicillin challenges.

Yuson et al ${ }^{64}$ diagnosed a hypersensitivity to the index cephalosporins in $24(46.3 \%)$ of 55 subjects with histories of cephalosporin immediate reactions. Among them, twenty-three subjects were ST negative to penicillin reagents; 7 of them underwent challenges with amoxicillin (6 subjects) or flucloxacillin (one subject) and tolerated them. The remaining subject was ST positive to amoxicillin and was not challenged.
Li et $\mathrm{al}^{69}$ assessed the safety and feasibility of amoxicillin challenges without penicillin STs in 40 patients with anaphylactic reactions to cefazolin and positive STs to it. This study ${ }^{69}$ also included 2 patients with cephalothin anaphylaxis, and 2 with ceftriaxone anaphylaxis; all 4 patients had positive STs to the responsible cephalosporins and negative penicillin STs. All 44 patients underwent a 3-day amoxicillin challenge without reporting immediate reactions. One patient experienced a delayed benign rash at 24 hours and ceased amoxicillin.

\section{Selecting Carbapenems or Aztreonam}

A systematic review ${ }^{70}$ of all published data on children and adults reporting immediate reactions to penicillins and/or cephalosporins who were subsequently given a carbapenem showed that for patients with previous proven, suspected, or possible IgE-mediated cephalosporin reactions $(n=12)$, the incidence of any type of HSR to a carbapenem was $25 \%$ ( 3 of 12 ); this included 2 non-IgEmediated reactions and 1 possible IgE-mediated reaction.

In an aforementioned study concerning 98 cephalosporin-allergic subjects, ${ }^{68} 1$ participant was ST positive to both meropenem and imipenem/cilastatin, as well as to all the other reagents tested, which indicates a sensitivity to an antigenic determinant of the $\beta$-lactam ring, and 3 subjects were positive to aztreonam: the one just mentioned, another with positive allergy test results also to cefodizime and PV, and the last with positive STs to both aztreonam and ceftazidime, the responsible drugs. Of note, the other 10 subjects who were allergic to ceftazidime were ST negative to aztreonam. In this study, ${ }^{68}$ all subjects tolerated the alternative $\beta$ lactams concerned found negative in skin testing, with the exception of 1 subject who reacted to imipenem/cilastatin.

\section{Selecting Alternative Cephalosporins}

There are few studies in which at least 5 patients with cephalosporin allergy were challenged with alternative cephalosporin found negative to STs.

A study ${ }^{71}$ evaluated 102 adults with immediate reactions to cephalosporins and positive STs to the responsible drugs by performing cefaclor sIgE assays and STs with a panel of 11 cephalosporins. On the basis of the results of both allergy tests, subjects were classified into four groups: group A (73 subjects), positive to one or more of ceftriaxone, cefuroxime, cefotaxime, cefepime, cefodizime, and ceftazidime; group B (13 subjects), positive to aminocephalosporins; group C (7 patients), positive to cephalosporins other than those belonging to the aforementioned 
groups; and group D (9 participants), positive to cephalosporins belonging to two different groups. In group A, 41 subjects were positive only to the responsible cephalosporins and 32 presented a pattern of cross-reactivity. In group $\mathrm{B}, 11$ subjects were positive only to the culprit aminocephalosporins and 2 presented a pattern of cross-reactivity. Of the 7 subjects of group C, 6 were positive only to the responsible compound (5 to cefazolin and 1 to cefamandole), and the remaining subject, who had reacted to cefoperazone, was positive to both cefoperazone and cefamandole. Group D subjects displayed different patterns of positivity, most of which cannot be explained by either similar or identical side chains or by the common $\beta$-lactam ring. These cases suggest the possibility of coexisting sensitivities; therefore, the rate of positive allergy test responses to other cephalosporins is not associated only with the chemical similarities among side-chain determinants. In this study, ${ }^{71}$ group A subjects underwent challenges with cefaclor, cefazolin, and ceftibuten; group B participants with cefuroxime axetil, ceftriaxone, cefazolin, and ceftibuten; and group $\mathrm{C}$ and $\mathrm{D}$ subjects with some of the aforementioned cephalosporins selected on the basis of their patterns of positivity. A total of 323 challenges with alternative cephalosporins (ceftibuten in 101, cefazolin in 96, cefaclor in 82, and cefuroxime axetil and ceftriaxone in 22 subjects) were well tolerated. These data indicate that cephalosporin hypersensitivity is improbable to be a class hypersensitivity. In effect, 2 groups (or subclasses) of cephalosporins were identified: group A, which includes those with a methoxyimino group in their R1 side chains plus ceftazidime, whose R1 side chain does not have a methoxyimino group but instead has an alkoxyimino group (Figure 2A and B), and group B, which is composed of aminocephalosporins. The limited number of subjects sensitive to cephalosporins other than those belonging to the aforementioned groups did not allow to identify further groups. However, based on the case of a group $\mathrm{C}$ subject who had reacted to cefoperazone and was ST positive to both cefoperazone and cefamandole, one could hypothesize additional groups, such as one consisting of cephalosporins like cefamandole, cefoperazone, and cefotetan that share an identical R2 side chain with an $\mathrm{N}$-methyltetrazole-thiol group (Figure 2A and B).

Subsequently, in a study by Sadleir et al, ${ }^{72} 21$ subjects diagnosed with immediate hypersensitivity to cefazolin, including 19 subjects with confirmed anaphylaxis, were negative to IDTs with cephalothin and tolerated challenges with it. Van Gasse et $\mathrm{al}^{73}$ administered ceftazidime to 5 patients who had experienced immediate HSRs to cefuroxime and displayed positive STs to cefuroxime and negative ones to ceftazidime. All subjects tolerated challenges with ceftazidime. This study ${ }^{73}$ proved that small structural dissimilarities might result in a lack of cross-reactivity and clinical tolerance. In a study by Stone et al, ${ }^{65}$ among 22 patients with a confirmed immediate allergy to either cefazolin, cefuroxime, ceftriaxone, ceftazidime, or cefepime, 17 tolerated an oral challenge with cephalexin, which has a R1 side chain different from those of the responsible cephalosporins. Another patient with a confirmed cefazolin allergy tolerated a cefuroxime challenge.

However, even though literature data ${ }^{9-12,60,61,65,74}$ indicate that cross-reactivity among cephalosporins is mainly connected with their R1 side chains, cases of crossreactivity related to the $\mathrm{R} 2$ side chain, such as the one observed in the aforementioned study of ours, ${ }^{71}$ are possible. $^{75}$

The above studies ${ }^{65,71-73}$ demonstrated the usefulness of considering the antigenic determinants of both $\mathrm{R} 1$ and R2 side chains when selecting alternative cephalosporins in cephalosporin-allergic subjects and the capability of STs to detect fine structural differences among cephalosporins in allergic subjects. In effect, a total of 367 challenges were performed with alternative cephalosporins found negative to STs, and none caused symptoms. Therefore, cephalosporin STs appear to be reliable and effective for selecting alternative cephalosporins in cephalosporinallergic subjects. In a large Korean study, ${ }^{76}$ however, routine screening IDTs with cephalosporins before administration of the cephalosporin concerned was not clinically useful for the prevention of anaphylaxis and related mortality.

Regarding individuals with a delayed allergy to cephalosporins, in the above study, ${ }^{65}$ among 7 patients with such allergy to either cefazolin, cefoxitin, ceftriaxone, or cefepime, 2 underwent an oral challenge with cephalexin and both tolerated it. Concerning subjects with SCARs, in the aforementioned study by Bérot et al, ${ }^{45}$ of the 3 subjects who had experienced a DRESS from cefoxitin, cefuroxime, and ceftriaxone, respectively, one presented a selective response to PT with cefoxitin, another was positive to cefuroxime, as well as to ceftriaxone and penicillins, and the last was positive to ceftriaxone, cefuroxime, ceftazidime, cefotaxime, and cefoxitin. In this study, ${ }^{45}$ however, subjects displaying negative results in allergy tests with cephalosporins other than those responsible did not undergo challenges with them. 


\section{Conclusions}

In choosing an alternative $\beta$-lactam for a $\beta$-lactam-allergic patient, it is important to consider its potential crossreactivity to the responsible drug.

The literature data ${ }^{9-12}$ indicate that similarities or identities of $\beta$-lactam side-chain structures are the main responsible for cross-reactivity among these antibiotics. In particular, the similarity or identity of the branch chain moiety of cephalosporin R1 structure is more frequently connected with cross-reactivity among cephalosporins than the similarity or identity of the ring of the R1 structure. ${ }^{9,10,76}$

Instead, the cross-reactivity related to the common $\beta$-lactam ring, which entails positive responses to all $\beta$ lactams tested, is very rare in subjects with an IgEmediated allergy and appears to be even rarer or absent in those with a T-cell-mediated allergy. ${ }^{9}$ Notably, in 3 studies, ${ }^{33,49,68} 2$ subjects with an IgE-mediated allergy to penicillins and 1 to cephalosporins, respectively, were ST positive to all reagents tested, including carbapenems and aztreonam. These 3 subjects probably had IgE antibodies to an antigenic determinant of the common $\beta$-lactam ring. Therefore, unlike what has been believed so far, ${ }^{18,53,77}$ there appears to be an immunologic cross-reactivity related to the common $\beta$ lactam ring not only between penicillins and carbapenems but also between penicillins and the monobactam aztreonam, as well as between cephalosporins and both carbapenems and aztreonam, although it is very rare.

In some studies that assessed cross-reactivity among $\beta$ lactams, ${ }^{23,29,30,68,71}$ there were particular patterns of allergytest positivity which cannot be explained by either similar or identical side chains or by the common $\beta$-lactam ring. Such patterns seem to indicate the possibility of coexisting sensitivities to different $\beta$-lactams likely due to previous exposure to them. Because of this possibility or, much less frequently, of a sensitivity to an antigenic determinant of the $\beta$-lactam ring, an allergist dealing with a $\beta$-lactam-allergic patient who needs an alternative $\beta$-lactam should perform STs with this drug, even if it has a different side chain from that of the culprit drug; if ST results are negative, she/he can give the $\beta$ lactam concerned with a graded challenge.

If is not possible to perform a complete allergy workup, individuals who report immediate reactions to penicillins and have a pressing need for a cephalosporin or another alternative $\beta$-lactam can be evaluated by STs with cephalosporins (or carbapenems, or aztreonam) that do not share similar or identical side chains with the culprit penicillins, and, in case of negative results, can undergo graded challenges with the alternative $\beta$-lactam concerned. A similar approach can be chosen in patients with histories of cephalosporin allergy who need an alternative $\beta$-lactam, including another cephalosporin.

In patients with mild nonimmediate reactions to $\beta$ lactams who require an alternative $\beta$-lactam, if there's no time to wait for the delayed reading of pre-treatment STs, giving a full dose of a structurally non-related $\beta$-lactam under close surveillance can be considered.

\section{Abbreviations}

AGEP, acute generalized exanthematous pustulosis; DRESS, drug reaction (or rash) with eosinophilia and systemic symptoms; HSR, hypersensitivity reaction; IDT, intradermal test; MPE, maculopapular exanthema; MSUD, maximum single unit dose; PG, penicillin G (or benzylpenicillin); PT, patch test; PV, penicillin V (or phenoxymethylpenicillin); PPL, benzylpenicilloyl-poly-L-lysine; SCAR, severe cutaneous adverse reaction; sIgE, serum specific IgE; SJS, Stevens-Johnson syndrome; ST, skin test; TEN, toxic epidermal necrolysis.

\section{Disclosure}

All authors declare no personal or professional conflicts of interest for this work.

\section{References}

1. Torres MJ, Blanca M. The complex clinical picture of beta-lactam hypersensitivity: penicillins, cephalosporins, monobactams, carbapenems, and clavams. Med Clin North Am. 2010;94(4):805-xii. doi:10.1016/j.mena.2010.04.006

2. Romano A, Torres MJ, Castells M, Sanz ML, Blanca M. Diagnosis and management of drug hypersensitivity reactions. J Allergy Clin Immunol. 2011;127(3 Suppl):S67-S73. doi:10.1016/j.jaci.2010.11.047

3. Demoly P, Adkinson NF, Brockow K, et al. International consensus on drug allergy. Allergy. 2014;69(4):420-437. doi:10.1111/all.12350

4. Romano A, Atanasković-Marković M, Barbaud A, et al. Towards a more precise diagnosis of hypersensitivity to beta-lactams - an EAACI position paper. Allergy. 2020;75(6):1300-1315. doi:10.1111/ all.14122

5. Pichler WJ. Immune pathomechanism and classification of drug hypersensitivity. Allergy. 2019;74(8):1457-1471. doi:10.1111/all.13 765

6. Romano A, Blanca M, Torres MJ, et al. Diagnosis of nonimmediate reactions to beta-lactam antibiotics. Allergy. 2004;59(11):1153-1160. doi:10.1111/j.1398-9995.2004.00678.x

7. Pichichero ME, Zagursky R. Penicillin and cephalosporin allergy. Ann Allergy Asthma Immunol. 2014;112(5):404-412. doi:10.1016/j. anai.2014.02.005

8. Mirakian R, Leech SC, Krishna MT, et al. Management of allergy to penicillins and other beta-lactams. Clin Exp Allergy. 2015;45 (2):300-327. doi:10.1111/cea.12468 
9. Romano A, Gaeta F, Arribas Poves MF, Valluzzi RL. Cross-reactivity among beta-lactams. Curr Allergy Asthma Rep. 2016;16(3):24. doi:10.1007/s11882-016-0594-9

10. Zagursky RJ, Pichichero ME. Cross-reactivity in $\beta$-lactam allergy. J Allergy Clin Immunol Pract. 2018;6(1):72-81.e1. doi:10.1016/j. jaip.2017.08.027

11. Chaudhry SB, Veve MP, Wagner JL. Cephalosporins: a focus on side chains and $\beta$-lactam cross-reactivity. Pharmacy (Basel). 2019;7 (3):103. doi:10.3390/pharmacy 7030103

12. Picard M, Robitaille G, Karam F, et al. Cross-reactivity to cephalosporins and carbapenems in penicillin-allergic patients: two systematic reviews and meta-analyses. J Allergy Clin Immunol Pract. 2019;7 (8):2722-2738.e5. doi:10.1016/j.jaip.2019.05.038

13. Blanca M, Romano A, Torres MJ, et al. Update on the evaluation of hypersensitivity reactions to betalactams. Allergy. 2009;64 (2):183-193. doi:10.1111/j.1398-9995.2008.01924.x

14. Brockow K, Romano A, Blanca M, Ring J, Pichler W, Demoly P. General considerations for skin test procedures in the diagnosis of drug hypersensitivity. Allergy. 2002;57(1):45-51.

15. Picard M, Bégin P, Bouchard $\mathrm{H}$, et al. Treatment of patients with a history of penicillin allergy in a large tertiary-care academic hospital. J Allergy Clin Immunol Pract. 2013;1(3):252-257. doi:10. 1016/j.jaip.2013.01.006

16. Macy E, Contreras R. Health care use and serious infection prevalence associated with penicillin "allergy" in hospitalized patients: a cohort study. J Allergy Clin Immunol. 2014;133(3):790-796. doi:10.1016/j.jaci.2013.09.021

17. Rubin R. Overdiagnosis of penicillin allergy leads to costly, inappropriate treatment. JAMA. 2018;320(18):1846-1848. doi:10.1001/ jama.2018.14358

18. Castells M, Khan DA, Phillips EJ, Longo DL. Penicillin allergy. $N$ Engl J Med. 2019;381(24):2338-2351. doi:10.1056/NEJMra18 07761

19. Lang DM, Castells MC, Khan DA, Murphy AW, Penicillin Allergy in Antibiotic Resistance Workgroup. Penicillin allergy testing should be performed routinely in patients with self-reported penicillin allergy. J Allergy Clin Immunol Pract. 2017;5(2):333-334. doi:10.1016/j. jaip.2016.12.010

20. Tanno LK, Torres MJ, Castells M, Demoly P, Joint Allergy Academies. What can we learn in drug allergy management from World Health Organization's international classifications? Allergy. 2018;73(5):987-992. doi:10.1111/all.13335

21. Blanca-Lopez N, Perez-Alzate D, Ruano F, et al. Selective immediate responders to amoxicillin and clavulanic acid tolerate penicillin derivative administration after confirming the diagnosis. Allergy. 2015;70 (8):1013-1019. doi:10.1111/all.12636

22. Romano A, Di Fonso M, Papa G, et al. Evaluation of adverse cutaneous reactions to aminopenicillins with emphasis on those manifested by maculopapular rashes. Allergy. 1995;50(2):113-118. doi:10.1111/j.1398-9995.1995.tb05066.x

23. Trcka J, Seitz CS, Bröcker EB, Gross GE, Trautmann A. Aminopenicillin-induced exanthema allows treatment with certain cephalosporins or phenoxymethyl penicillin. $J$ Antimicrob Chemother. 2007;60(1):107-111. doi:10.1093/jac/dkm146

24. Kennard L, Rutkowski K, Siew LQC, et al. Flucloxacillin hypersensitivity: patient outcomes in a multicenter retrospective study. J Allergy Clin Immunol Pract. 2019;7(7):2212-2217.e1. doi:10.10 16/j.jaip.2019.03.018

25. Pichichero ME. A review of evidence supporting the American academy of pediatrics recommendation for prescribing cephalosporin antibiotics for penicillin-allergic patients. Pediatrics. 2005;115 (4):1048-1057. doi:10.1542/peds.2004-1276

26. Pichichero ME, Casey JR. Safe use of selected cephalosporins in penicillin-allergic patients: a meta-analysis. Otolaryngol Head Neck Surg. 2007;136(3):340-347. doi:10.1016/j.otohns.2006.10.007
27. Audicana M, Bernaola G, Urrutia I, et al. Allergic reactions to betalactams: studies in a group of patients allergic to penicillin and evaluation of cross-reactivity with cephalosporin. Allergy. 1994;49 (2):108-113. doi:10.1111/j.1398-9995.1994.tb00809.x

28. Novalbos A, Sastre J, Cuesta J, et al. Lack of allergic cross-reactivity to cephalosporins among patients allergic to penicillins. Clin Exp Allergy. 2001;31(3):438-443. doi:10.1046/j.1365-2222.2001.00992.x

29. Romano A, Guéant-Rodriguez RM, Viola M, Pettinato R, Guéant JL. Cross-reactivity and tolerability of cephalosporins in patients with immediate hypersensitivity to penicillins. Ann Intern Med. 2004;141 (1):16-22. doi:10.7326/0003-4819-141-1-200407060-00010

30. Caimmi S, Galéra C, Bousquet-Rouanet L, Arnoux B, Demoly P, Bousquet PJ. Safety of cefuroxime as an alternative in patients with a proven hypersensitivity to penicillins: a DAHD cohort survey. Int Arch Allergy Immunol. 2010;153(1):53-60. doi:10.1159/00030 1579

31. Romano A, Valluzzi RL, Caruso C, Maggioletti M, Quaratino D, Gaeta F. Cross-reactivity and tolerability of cephalosporins in patients with ige-mediated hypersensitivity to penicillins. $J$ Allergy Clin Immunol Pract. 2018;6(5):1662-1672. doi:10.1016/j.jaip.2018.01.020

32. Rodriguez-Bouza T, Al-Ahmad M. Drug allergy evaluation for betalactam hypersensitivity: cross-reactivity with cephalosporines, carbapenems and negative predictive value. Asian Pac J Allergy Immunol. 2018;36(1):27-31. doi:10.12932/AP0853

33. Romano A, Valluzzi RL, Caruso C, Zaffiro A, Quaratino D, Gaeta F. Tolerability of cefazolin and ceftibuten in patients with ige-mediated aminopenicillin allergy. J Allergy Clin Immunol Pract. 2020;8 (6):1989-1993.e2. doi:10.1016/j.jaip.2020.02.025

34. Sánchez de Vicente J, Gamboa P, García-Lirio E. Tolerance to cephalosporins and carbapenems in penicillin-allergic patients. J Investig Allergol Clin Immunol. 2020;30(1):75-76. doi:10.18176/ jiaci.0463

35. Blanca M, Fernandez J, Miranda A, et al. Cross-reactivity between penicillins and cephalosporins: clinical and immunologic studies. $J$ Allergy Clin Immunol. 1989;83(2 Pt 1):381-385. doi:10.1016/ 0091-6749(89)90122-X

36. Sastre J, Quijano LD, Novalbos A, et al. Clinical cross-reactivity between amoxicillin and cephadroxil in patients allergic to amoxicillin and with good tolerance of penicillin. Allergy. 1996;51 (6):383-386. doi:10.1111/j.1398-9995.1996.tb00146.x

37. Miranda A, Blanca M, Vega JM, et al. Cross-reactivity between a penicillin and a cephalosporin with the same side chain. J Allergy Clin Immunol. 1996;98(3):671-677. doi:10.1016/S0091-6749(96) 70101-X

38. Park MA, Koch CA, Klemawesch P, Joshi A, Li JT. Increased adverse drug reactions to cephalosporins in penicillin allergy patients with positive penicillin skin test. Int Arch Allergy Immunol. 2010;153 (3):268-273. doi:10.1159/000314367

39. Macy E, Blumenthal KG. Are cephalosporins safe for use in penicillin allergy without prior allergy evaluation? J Allergy Clin Immunol Pract. 2018;6(1):82-89. doi:10.1016/j.jaip.2017.07.033

40. Phillips E, Knowles SR, Weber EA, Blackburn D. Cephalexin tolerated despite delayed aminopenicillin reactions. Allergy. 2001;56 (8):790. doi:10.1034/j.1398-9995.2001.056008790.x

41. Romano A, Gaeta F, Valluzzi RL, Maggioletti M, Caruso C, Quaratino D. Cross-reactivity and tolerability of aztreonam and cephalosporins in subjects with a $\mathrm{T}$ cell-mediated hypersensitivity to penicillins. $J$ Allergy Clin Immunol. 2016;138(1):179-186. doi:10.1016/j.jaci.2016.01.025

42. Joint Task Force on Practice Parameters; American Academy of Allergy, Asthma and Immunology; American College of Allergy, Asthma and Immunology; Joint Council of Allergy, Asthma and Immunology. Drug allergy: an updated practice parameter. Ann Allergy Asthma Immunol. 2010;105(4):259-273. doi:10.1016/j. anai.2010.08.002 
43. Blumenthal KG, Youngster I, Shenoy ES, Banerji A, Nelson SB Tolerability of cefazolin after immune-mediated hypersensitivity reactions to nafcillin in the outpatient setting. Antimicrob Agents Chemother. 2014;58(6):3137-3143. doi:10.1128/AAC.02504-13

44. Trubiano JA, Chua KYL, Holmes NE, et al. Safety of cephalosporins in penicillin class severe delayed hypersensitivity reactions. J Allergy Clin Immunol Pract. 2020;8(3):1142-1146.e4. doi:10.1016/j.jaip.20 19.10.005

45. Bérot V, Gener G, Ingen-Housz-Oro S, et al. Cross-reactivity in beta-lactams after a non-immediate cutaneous adverse reaction: experience of a reference centre for toxic bullous diseases and severe cutaneous adverse reactions. J Eur Acad Dermatol Venereol. 2020;34 (4):787-794. doi:10.1111/jdv.15986

46. Lieberman P, Kemp S, Oppenheimer J, Joint Task Force on Practice Parameters; American Academy of Allergy, Asthma and Immunology; American College of Allergy, Asthma and Immunology; Joint Council of Allergy, Asthma and Immunology. The diagnosis and management of anaphylaxis: an updated practice parameter. J Allergy Clin Immunol. 2005;115(3 Suppl 2):S483-S523. doi:10.1016/j.jaci.2005.01.010

47. Saxon A, Adelman DC, Patel A, Hajdu R, Calandra GB. Imipenem cross-reactivity with penicillin in humans. $J$ Allergy Clin Immunol. 1988;82(2):213-217. doi:10.1016/0091-6749(88)91001-9

48. Romano A, Viola M, Guéant-Rodriguez RM, Gaeta F, Pettinato R, Guéant JL. Imipenem in patients with immediate hypersensitivity to penicillins. $N$ Engl J Med. 2006;354(26):2835-2837. doi:10.1056/ NEJMc053529

49. Romano A, Viola M, Guéant-Rodriguez RM, Gaeta F, Valluzzi R, Guéant JL. Brief communication: tolerability of meropenem in patients with IgE-mediated hypersensitivity to penicillins. Ann Intern Med. 2007;146(4):266-269. doi:10.7326/0003-4819-1464-200702200-00005

50. Atanasković-Marković M, Gaeta F, Medjo B, Viola M, Nestorović B, Romano A. Tolerability of meropenem in children with IgE-mediated hypersensitivity to penicillins. Allergy. 2008;63(2):237-240. doi:10. 1111/j.1398-9995.2007.01532.x

51. Atanasković-Marković M, Gaeta F, Gavrović-Jankulović M, Veličković TC, Valluzzi RL, Romano A. Tolerability of imipenem in children with IgE-mediated hypersensitivity to penicillins. $J$ Allergy Clin Immunol. 2009;124(1):167-169. doi:10.1016/j. jaci.2009.02.031

52. Gaeta F, Valluzzi RL, Alonzi C, Maggioletti M, Caruso C, Romano A. Tolerability of aztreonam and carbapenems in patients with IgE-mediated hypersensitivity to penicillins. J Allergy Clin Immunol. 2015;135(4):972-976. doi:10.1016/j.jaci.2014.10.011

53. Shenoy ES, Macy E, Rowe T, Blumenthal KG. Evaluation and management of penicillin allergy: a review. JAMA. 2019;321 (2):188-199. doi:10.1001/jama.2018.19283

54. Romano A, Gaeta F, Valluzzi RL, et al. Absence of cross-reactivity to carbapenems in patients with delayed hypersensitivity to penicillins. Allergy. 2013;68(12):1618-1621. doi:10.1111/all.12299

55. Schiavino D, Nucera E, Lombardo C, et al. Cross-reactivity and tolerability of imipenem in patients with delayed-type, cell-mediated hypersensitivity to beta-lactams. Allergy. 2009;64 (11):1644-1648. doi:10.1111/j.1398-9995.2009.02058.x

56. Levine BB, Feingold DS, Parris EE. Immunologic mechanisms of penicillin allergy. A haptenic model system for the study of allergic diseases of man. $N$ Engl $J$ Med. 1966;275(20):1115-1125. doi:10.1056/NEJM196611172752009

57. Fernando SL. Ertapenem-induced acute generalized exanthematous pustulosis with cross-reactivity to other beta-lactam antibiotics on patch testing. Ann Allergy Asthma Immunol. 2013;111(2):139-140. doi:10.1016/j.anai.2013.05.015
58. Sameed M, Nwaiser C, Bhandari P, Schmalzle SA. Meropeneminduced Stevens-Johnson syndrome/toxic epidermal necrolysis in a patient with known type IV penicillin hypersensitivity. BMJ Case Rep. 2019;12(8):e230144. doi:10.1136/bcr-2019-230144

59. Khan DA, Banerji A, Bernstein JA, et al. Cephalosporin allergy: current understanding and future challenges. $J$ Allergy Clin Immunol Pract. 2019;7(7):2105-2114. doi:10.1016/j.jaip.2019.06.001

60. Romano A, Guéant-Rodriguez RM, Viola M, et al. Diagnosing immediate reactions to cephalosporins. Clin Exp Allergy. 2005;35 (9):1234-1242. doi:10.1111/j.1365-2222.2005.02317.x

61. Antunez C, Blanca-López N, Torres MJ, et al. Immediate allergic reactions to cephalosporins: evaluation of cross-reactivity with a panel of penicillins and cephalosporins. J Allergy Clin Immunol. 2006;117(2):404-410. doi:10.1016/j.jaci.2005.10.032

62. Romano A, Gaeta F, Valluzzi RL, Alonzi C, Viola M, Bousquet PJ. Diagnosing hypersensitivity reactions to cephalosporins in children. Pediatrics. 2008;122(3):521-527. doi:10.1542/peds.2007-3178

63. Mori F, Liccioli G, Piccorossi A, et al. The diagnosis of ceftriaxone hypersensitivity in a paediatric population. Int Arch Allergy Immunol. 2019;178(3):272-276. doi:10.1159/000495302

64. Yuson C, Kumar K, Le A, et al. Immediate cephalosporin allergy. Intern Med J. 2019;49(8):985-993. doi:10.1111/imj.14229

65. Stone CA Jr, Trubiano JA, Phillips EJ. Testing strategies and predictors for evaluating immediate and delayed reactions to cephalosporins [published online ahead of print, 2020 Aug 18]. J Allergy Clin Immunol Pract. 2020.

66. Romano A, Gaeta F, Valluzzi RL, et al. Diagnosing nonimmediate reactions to cephalosporins. J Allergy Clin Immunol. 2012;129 (4):1166-1169. doi:10.1016/j.jaci.2011.12.995

67. Yoon SY, Park SY, Kim S, et al. Validation of the cephalosporin intradermal skin test for predicting immediate hypersensitivity: a prospective study with drug challenge. Allergy. 2013;68 (7):938-944. doi:10.1111/all.12182

68. Romano A, Gaeta F, Valluzzi RL, Caruso C, Rumi G, Bousquet PJ. IgE-mediated hypersensitivity to cephalosporins: cross-reactivity and tolerability of penicillins, monobactams, and carbapenems. $J$ Allergy Clin Immunol. 2010;126(5):994-999. doi:10.1016/j. jaci.2010.06.052

69. Li J, Green SL, Krupowicz BA, et al. Cross-reactivity to penicillins in cephalosporin anaphylaxis. Br J Anaesth. 2019;123(6):e532-e534. doi:10.1016/j.bja.2019.09.011

70. Kula B, Djordjevic G, Robinson JL. A systematic review: can one prescribe carbapenems to patients with IgE-mediated allergy to penicillins or cephalosporins? Clin Infect Dis. 2014;59(8):1113-1122. doi:10.1093/cid/ciu587

71. Romano A, Gaeta F, Valluzzi RL, et al. IgE-mediated hypersensitivity to cephalosporins: cross-reactivity and tolerability of alternative cephalosporins. J Allergy Clin Immunol. 2015;136(3):685-691.e3. doi:10.1016/j.jaci.2015.03.012

72. Sadleir PH, Clarke RC, Platt PR. Cefalotin as antimicrobial prophylaxis in patients with known intraoperative anaphylaxis to cefazolin. Br J Anaesth. 2016;117(4):464-469. doi:10.1093/bja/aew274

73. Van Gasse AL, Ebo DG, Faber MA, et al. Cross-reactivity in IgE-mediated allergy to cefuroxime: focus on the R1 side chain. J Allergy Clin Immunol Pract. 2020;8(3):1094-1096.e1. doi:10. 1016/j.jaip.2019.08.033

74. Romano A, Mayorga C, Torres MJ, et al. Immediate allergic reactions to cephalosporins: cross-reactivity and selective responses. $J$ Allergy Clin Immunol. 2000;106(6):1177-1183. doi:10.1067/ mai.2000.111147

75. Baldo BA, Pham NH. Allergenic significance of cephalosporin side chains. J Allergy Clin Immunol. 2015;136(5):1426-1428. doi:10. 1016/j.jaci.2015.06.051 
76. Yang MS, Kang DY, Seo B, et al. Incidence of cephalosporin-induced anaphylaxis and clinical efficacy of screening intradermal tests with cephalosporins: a large multicenter retrospective cohort study. Allergy. 2018;73(9):1833-1841. doi:10.1111/all.13435
77. Har D, Solensky R. Penicillin and beta-lactam hypersensitivity. Immunol Allergy Clin North Am. 2017;37(4):643-662. doi:10.1016/ j.iac.2017.07.001

\section{Publish your work in this journal}

The Journal of Asthma and Allergy is an international, peer-reviewed open-access journal publishing original research, reports, editorials and commentaries on the following topics: Asthma; Pulmonary physiology; Asthma related clinical health; Clinical immunology and the immunological basis of disease; Pharmacological interventions and

Submit your manuscript here: https://www.dovepress.com/journal-of-asthma-and-allergy-journal new therapies. The manuscript management system is completely online and includes a very quick and fair peer-review system, which is all easy to use. Visit http://www.dovepress.com/testimonials.php to read real quotes from published authors. 